University of Nebraska - Lincoln

DigitalCommons@University of Nebraska - Lincoln

Faculty Publications, Department of Mathematics

Mathematics, Department of

4-1968

A PROOF OF NEWTON'S POWER SUM FORMULAS

J. A. Eidswick

Follow this and additional works at: https://digitalcommons.unl.edu/mathfacpub

Part of the Mathematics Commons

Eidswick, J. A., "A PROOF OF NEWTON'S POWER SUM FORMULAS" (1968). Faculty Publications, Department of Mathematics. 50.

https://digitalcommons.unl.edu/mathfacpub/50

This Article is brought to you for free and open access by the Mathematics, Department of at DigitalCommons@University of Nebraska - Lincoln. It has been accepted for inclusion in Faculty Publications, Department of Mathematics by an authorized administrator of DigitalCommons@University of Nebraska - Lincoln. 


\section{A PROOF OF NEWTON'S POWER SUM FORMULAS}

J. A. EIDSwICK, University of Nebraska

For a polynomial $P(z)=\alpha_{0}+\alpha_{1} z+\cdots+\alpha_{n} z^{n}=\alpha_{n}\left(z-z_{1}\right)\left(z-z_{2}\right) \cdots\left(z-z_{n}\right)$, the power sums $S_{m}=\sum_{k=1}^{n} z_{k}^{m}, m=1,2, \cdots$, can be calculated from the formulas

$$
m \alpha_{n-m}+\sum_{k=1}^{m} \alpha_{n-m+k} S_{k}=0 \text { if } m \leqq n
$$

$$
\sum_{k=m-n}^{m} \alpha_{n-m+k} S_{k}=0 \text { if } m>n \text {. }
$$

For example, if $n=3$,

$$
\begin{aligned}
& S_{1}=-\alpha_{2} \alpha_{3}^{-1}, \quad S_{2}=\alpha_{2}^{2} \alpha_{3}^{-2}-2 \alpha_{1} \alpha_{3}^{-1}, \quad S_{3}=-\alpha_{2}^{3} \alpha_{3}^{-3}+3 \alpha_{1} \alpha_{2} \alpha_{3}^{-2}-3 \alpha_{0} \alpha_{3}^{-1}, \\
& S_{4}=\alpha_{2}^{4} \alpha_{3}^{-4}-4 \alpha_{1} \alpha_{2}^{2} \alpha_{3}^{-3}+4 \alpha_{0} \alpha_{2} \alpha_{3}^{-2}+2 \alpha_{1}^{2} \alpha_{3}^{-2} .
\end{aligned}
$$


One quick, but rather vague, method of proving (1) is to differentiate $P(z)$ two different ways, equating like powers of $z$ (see [1]). Another method is through the use of the theory of symmetric functions (see [2]). The student might find the following proof more satisfying: The logarithmic derivative of the polynomial $Q(z)=\alpha_{n}+\alpha_{n-1} z+\cdots+\alpha_{0} z^{n}=\alpha_{0}\left(z-z_{1}^{-1}\right)\left(z-z_{2}^{-1}\right) \cdots\left(z-z_{n}^{-1}\right) \quad$ (assuming, without loss of generality, that $P(0) \neq 0)$ is

$$
F(z)=\frac{Q^{\prime}(z)}{Q(z)}=\sum_{k=1}^{n}\left(z-z_{k}^{-1}\right)^{-1}
$$

which, when differentiated $k$ times, gives $F^{(k)}(0)=-k ! S_{k+1}$. Since

$$
Q^{(m)}(z)=[F(z) Q(z)]^{(m-1)}=\sum_{k=0}^{m-1}\left(\begin{array}{c}
m-1 \\
k
\end{array}\right) F^{(k)}(z) Q^{(m-1-k)}(z),
$$

we have

$$
\frac{Q^{(m)}(0)}{m !}=-\frac{1}{m} \sum_{k=0}^{m-1} \frac{Q^{(m-1-k)}(0)}{(m-1-k) !} S_{k+1}
$$

or

$$
\begin{aligned}
-m \alpha_{n-m} & =\sum_{k=0}^{m-1} \alpha_{n-m+k+1} S_{k+1} & \text { if } m \leqq n, \\
0 & =\sum_{k=m-n-1}^{m-1} \alpha_{n-m+k+1} S_{k+1} & \text { if } m>n,
\end{aligned}
$$

which is (1).

\section{References}

1. L. E. Dickson, Linear Groups, B. G. Teubner, Stuttgart, 1901, p. 53.

2. B. L. van der Waerden, Modern Algebra, Frederick Ungar, New York, 1953. 